\title{
Efeitos adversos do tratamento com inibidores seletivos da recaptação da serotonina sobre o feto e neonatais de mães com depressão gestacional: Uma revisão crítica
}

\author{
Effects adverse of treatment with selective inhibitors of serotonin recaptation on the fetus and \\ neonatals of mothers with gestational depression: A critical review \\ Efectos adversos del tratamiento con inhibidores selectivos de la recaptación de serotonina en el \\ feto y neonatales de madres con depresión gestacional: Una revisión crítica
}

Recebido: 15/03/2021 | Revisado: 21/03/2021 | Aceito: 23/03/2021 | Publicado: 01/04/2021

\author{
Thais Pereira Nunes \\ ORCID: https://orcid.org/0000-0002-2446-7639 \\ Universidade Guarulhos, Brasil \\ Email: thais.p.nunes@hotmail.com \\ Larissa Pereira Nunes \\ ORCID: https://orcid.org/0000-0001-8322-5210 \\ Universidade Estadual Paulista, Brasil \\ Email: larissanunesp@hotmail.com \\ Arles Naisa Amaral Silva \\ ORCID: https://orcid.org/0000-0002-5265-161X \\ Universidade Estadual Paulista, Brasil \\ Email: arlesnaisa@hotmail.com \\ Beatriz Ommati Pirovani \\ ORCID: https://orcid.org/0000-0003-4536-6674 \\ Universidade Estadual Paulista, Brasil \\ Email: beatrizpirovani@gmail.com \\ Maria Juliana Sismeiro Dias Morábito \\ ORCID: https://orcid.org/0000-0002-8913-0914 \\ Universidade Estadual Paulista, Brasil \\ Email: maju_sismeiro@hotmail.com \\ Leonardo Antônio de Morais \\ ORCID: https://orcid.org/0000-0003-1894-0087 \\ Universidade Estadual Paulista, Brasil \\ Email: leo.a.morais@gmail.com \\ Amanda Scarpin Gruba \\ ORCID: https://orcid.org/0000-0003-4798-2972 \\ Universidade Estadual Paulista, Brasil \\ Email: amandascarpin97@gmail.com \\ Gabriel Pereira Nunes \\ ORCID: https://orcid.org/0000-0001-7001-967X \\ Universidade Estadual Paulista, Brasil \\ Email: gabriel.pereira.nunes@hotmail.com
}

\begin{abstract}
Resumo
A depressão é um dos transtornos psiquiátricos mais comuns, tendo em vista a alta incidência nesta década, nota-se um predomínio maior de ocorrência em mulheres, destacando o transtorno depressivo maior na gestação. Quando diagnosticada, o tratamento é de acordo o nível da doença, e se tratando de nível moderado a grave inicia-se o tratamento farmacológico com antidepressivos. Os medicamentos mais prescritos são os inibidores seletivos da receptação de Serotonina (ISRS), que tornaram-se fármacos de primeira escolha pela maior segurança em relação aos demais. Entretanto, o tratamento com os ISRS durante a gestação tem implicado em efeitos indesejáveis nos fetos e neonatos. Portanto, o objetivo deste trabalho foi investigar os efeitos adversos sobre o feto/neonatais de gestantes depressivas que passaram por terapêutica com ISRS. Este estudo caracterizou-se em uma revisão crítica, sendo utilizados os bancos de dados da Pubmed/MEDLINE, Scopus e Web of Science, sendo incluídos 35 artigos. Após análise dos estudos, foi constatado que a terapia com ISRS na depressão gestacional aumenta os riscos no feto e neonatos para defeitos congênitos, como: má formação cardíaca, abstinência neonatal, hipertensão pulmonar persistente e possível associação com transtorno do espectro do autismo. Assim, baseado nas evidências científicas o protocolo medicamentoso com ISRS gera efeitos nocivos a criança, no entanto muitas vezes o seu uso na gestação é inevitável. Dessa forma, em situações em que é necessário usar ISRS, a decisão de tal conduta terapêutica deve ser avaliada analisando os riscos da depressão não tratada e a relação risco/benefício do uso dos medicamentos.
\end{abstract}


Palavras-chave: Antidepressivos; ISRSs; Riscos; Depressão gestacional; Tratamento.

\begin{abstract}
Depression is one of the most common psychiatric disorders, due to the high incidence in this decade, a greater predominance of occurrence in women, among them the major depressive disorder in pregnancy. When the diagnosis is made, the treatment is according to the level of the disease, when it comes to a moderate to severe level, pharmacological treatment with antidepressants begins. Among them, the most prescribed are selective serotonin reuptake inhibitors (SSRIs), they have become first-line drugs of choice because they have a higher safety than the others. However, treatment with SSRIs during pregnancy has implicated several undesirable effects in fetuses and neonates. Therefore, the objective of this study was to investigate the adverse effects on the fetus / neonates of depressive women who underwent drug therapy with SSRIs. This study was characterized in a critical review, using the databases of Pubmed/MEDLINE, Scopus and Web of Science, in which 35 articles were included. After careful analysis of the studies, it was found that SSRI therapy in gestational depression increases the risks to the fetus and neonates for birth defects, such as: cardiac malformation, neonatal abstinence, persistent pulmonary hypertension and possible association with autism spectrum disorder. Therefore, based on scientific evidence, the SSRI drug protocol generates harmful effects on the child, however, the use of antidepressants during pregnancy is often inevitable. Thus, in cases where it may be necessary to use SSRIs, a decision on such a therapeutic approach should be evaluated by analyzing the risks of untreated depression and the risk / benefit ratio of the use of medications.
\end{abstract}

keywords: Antidepressants; SSRIs; Risks; Gestational depression; Treatment.

\title{
Resumen
}

La depresión es uno de los trastornos psiquiátricos más comunes, dada la alta incidencia en esta década, existe una mayor prevalencia de ocurrencia en mujeres, entre las que destaca el trastorno depresivo mayor en el embarazo. Cuando se hace el diagnóstico, el tratamiento es según el nivel de la enfermedad, cuando es de moderada a grave, se inicia el tratamiento farmacológico con antidepresivos. Entre ellos, los más recetados son los inhibidores selectivos de la recepción de serotonina (ISRS), que se han convertido en fármacos de primera elección porque son más seguros que los demás. Sin embargo, el tratamiento con ISRS durante el embarazo ha implicado varios efectos indeseables en fetos y recién nacidos. Por lo tanto, el objetivo de este estudio fue investigar los efectos adversos en el feto / recién nacidos de mujeres embarazadas deprimidas que se sometieron a terapia con fármacos ISRS. Este estudio se caracterizó en una revisión crítica, utilizando las bases de datos de Pubmed/MEDLINE, Scopus y Web of Science, en la que se incluyeron 35 artículos. Después de un análisis cuidadoso de los estudios, se encontró que la terapia con ISRS en la depresión gestacional aumenta los riesgos en el feto y los recién nacidos de defectos congénitos, tales como: malformación cardíaca, abstinencia neonatal, hipertensión pulmonar persistente y posible asociación con el trastorno del espectro autista. Por tanto, con base en la evidencia científica, el protocolo de fármacos ISRS genera efectos nocivos en el niño, sin embargo, el uso de antidepresivos durante el embarazo suele ser inevitable. Así, en situaciones en las que pueda ser necesario utilizar ISRS, la decisión de dicha conducta terapéutica debe evaluarse analizando los riesgos de depresión no tratada y la relación riesgo / beneficio del uso de medicamentos.

Palabras clave: Antidepresivos; ISRS; Riesgos; Depresión gestacional; Tratamiento.

\section{Introdução}

A depressão é um dos transtornos psiquiátricos mais comuns. Essa enfermidade é caracterizada pela deficiência dos neurotransmissores monoaminérgicos, principalmente noradrenalina e serotonina; dentro das subdivisões da síndrome da depressão a forma mais frequente é o Transtorno Depressivo Maior (TDM), os sintomas desse transtorno são definidos por mau humor persistente, perda de interesse e disposição. Essas manifestações afetam o desempenho e a qualidade de vida da pessoa acometida (Dubovicky et al., 2017).

Tendo em vista a alta incidência nesta década, nota-se um predomínio maior de ocorrência em mulheres que nos homens. Dentro dos transtornos psiquiátricos que acomete mulheres, destaca-se a o TDM no período gestacional. Esse transtorno na gestação e no puerpério são mais comuns do que se cogita, e durante a gravidez, até $20 \%$ das mulheres apresentam sintomas depressivos, e muitos casos ainda são sub diagnosticados (Zemestani, \& Fazeli Nikoo, 2020). Esses transtornos costumam acometer pacientes que já tenham história de patologia psiquiátrica prévia. Ao contrário do que se idealiza o período da gestação nem sempre é só marcado por alegrias e satisfações, uma vez que, muitas mulheres apresentam tristeza e ansiedade nessa etapa de suas vidas. A gestação é caracterizada como uma época delicada na vida da mulher, uma vez que envolve inúmeras alterações físicas, hormonais, psicológicas, e de inserção social, as quais podem refletir diretamente na sua saúde mental (Tang et al., 2019). 
Nos últimos anos tem-se dado importância crescente ao tema, e quando a depressão é diagnosticada no transcorrer da gestação, inicia-se o tratamento farmacológico de acordo o nível apresentado: moderado a grave. Pesquisas recentes têm focado também nos riscos que o tratamento com psicofármacos para essa patologia pode ocasionar não só à saúde da mãe, mas também ao desenvolvimento do feto, já que esses medicamentos atravessam a placenta facilmente (Alwa et al., 2016). Desta forma, gestantes que necessitam de tratamento para depressão e seus médicos muitas vezes enfrentam uma difícil escolha relacionando risco e benefícios do uso dos medicamentos. Embora não exista um antidepressivo que possa ser indicado para gestante com 100\% de segurança para o bebê, uns são mais adequados que outros (Zemestani, \& Fazeli Nikoo, 2020; Latendresse et al., 2017).

Dentre os fármacos prescritos, nota-se um período evolutivo quanto à administração. Em 1987, o tratamento da depressão foi revolucionado com a introdução dos Inibidores Seletivos da Receptação de Serotonina (ISRSs): citalopram, fluvoxamina, paroxetina, fluoxetina e sertralina, principalmente. Embora a eficácia desses medicamentos seja semelhante com outras classes de antidepressivos, eles tornaram-se agentes de primeira linha de escolha, com uma frequência de prescrição de 2,3\% em mulheres grávidas, por terem uma segurança e risco/benefício maior em relação aos demais. Contudo, apesar da maioria dos bebês nascidos de gestantes que faziam uso de ISRSs durante a gravidez sejam sadios, há indícios acumulados de que o tratamento materno com ISRS durante a gestação pode causar resultados reprodutivos adversos (Latendresse et al., 2017; Yazdy et al., 2014).

O tratamento com ISRSs na depressão durante a gestação tem sido implicado no aumento dos riscos de defeitos congênitos, aparentemente aumentam a taxa de malformações cardíacas, várias complicações neonatais, incluindo sintomas de abstinência neonatal e toxicidade, prematuridade, baixo peso, aumento da taxa de persistência da hipertensão pulmonar do recémnascido (Dubovicky et al., 2017; Alwa et al., 2016). Além dsso, há achados recentes sobre a possível associação de ISRSs com o transtorno do espectro do autismo (Latendresse et al., 2017).

Considerando a ocorrência de vários casos de depressão na gestação, é de grande relevância a identificação dos possíveis riscos que a terapêutica medicamentosa com ISRS expõe o feto e neonato, uma vez que essa classe é considerada como primeira linha de escolha na intervenção farmacológica, levando em consideração que o assunto é bastante abordado, pouco pesquisado e gera muitas dúvidas, parece oportuno analisar as pesquisas já existentes, investigando e esclarecendo os fatores relacionados ao tratamento e os riscos que o bebe está sendo exposto quando a gestante adere essa medicação. Pensando dessa foram, o objetivo deste trabalho foi investigar os efeitos adversos sobre o feto / neonatais de gestantes depressivas que passaram por terapêutica medicamentosa com antidepressivos inibidores seletivos da recaptação de serotonina (ISRS).

\section{Metodologia}

Considerando o objetivo proposto, essa pesquisa caracterizou-se em uma revisão crítica, modelo qualitativo (Pereira et al., 2018; Ludke \& Andre, 2013). A busca foi realizada no período de novembro de 2018 a fevereiro de 2019, sendo utilizados os bancos de dados da Pubmed/MEDLINE, SCOPUS e Web of Science. A seleção dos artigos seguiu os seguintes critérios de inclusão: artigos publicados em inglês, textos disponíveis nas bases de dados selecionadas e abordagem do tema da pesquisa. Como critérios de exclusão: artigos não disponíveis na íntegra no momento da pesquisa e que não apresentassem abordagem do tema no contexto de terapêutica da depressão gestacional com ISRSs e desfechos relacionados aos efeitos sobre feto e neonatais. As palavras usadas para estratégia de busca usada foram: (DeCS/MeSH): "Inibidores de Captação de Serotonina (Serotonin Uptake Inhibitors)", "Depressão (Depression)", "Gestantes (Pregnant)", “Antidepressivos (Antidepressives agents)” e "Bebê (Infant)","Efeitos Colaterais e reações adversas relacionadas a Medicamentos (Drug-related side effects and adverse reactions)" pesquisados juntos e encontrados no título dos artigos em todas as bases de dados. Estabelecendo avaliação criteriosa foram incluídos 35 artigos. 


\section{Revisão Crítica}

\subsection{Depressão: conceito e fisiopatologia}

A depressão é um dos distúrbios de saúde mental mais comum no mundo e acompanha a humanidade por toda a sua história. Considerada pela Organização Mundial da Saúde (OMS) como o "Mal do Século", é um problema afetivo que afeta o emocional da pessoa. Esse transtorno é reconhecido pela deficiência dos neurotransmissores monoaminérgicos (Smith, 2014). Os sistemas monoaminérgicos são compostos por neurônios que contêm norepinefrina (NE), serotonina (5-HT) e dopamina (DA). Junto com a acetilcolina (ACh), eles exercem efeitos de modulação e integração sobre outras atividades corticais e subcorticais e estão envolvidos na regulação da atividade psicomotora, apetite, sono e, provavelmente, do humor (Oyebode, 2012).

Propõe-se que a Depressão estaria relacionada ao funcionamento bioquímico inadequado da atividade de neurotransmissores, notadamente da serotonina, noradrenalina e dopamina (Alwa et al., 2016; Smith, 2014). E de fato, a hipótese de hipofuncionamento dos sistemas de neurotransmissores ganhou mais credibilidade depois que alguns antidepressivos agiam aumentando esses neurotransmissores e, concomitantemente, melhorando os sintomas da depressão. Entretanto, estas hipóteses não explicavam a falta de eficácia imediata dos tratamentos antidepressivos, apesar desses medicamentos aumentarem as concentrações sinápticas de serotonina e de noradrenalina quase imediatamente após sua ingestão. Apesar dessas novas e esperançosas hipóteses, vários neurotransmissores (serotonina, noradrenalina, dopamina, GABA, acetilcolina) e neuropeptídios (somatostatina, vasopresina, colecistocinina, opióides endógenos, entre outros) continuam se relacionando atualmente, de uma forma direta ou indireta, na patogênese dos Transtornos Afetivos.

A forma mais comum dessa enfermidade é o transtorno depressivo maior. Uma síndrome caracterizada por alteração da capacidade do indivíduo realizar suas atividades normais. Por apresentar tristeza profunda, falta de apetite, de ânimo, perda de interesse generalizado, sentimento de culpa, angustia crises de choro, déficit de memória, isolamento social, pensamento suicida e aumento de irritabilidade, essas manifestações ocasiona forte impacto na qualidade de vida da pessoa afetada ${ }^{7}$. A depressão acontece em meio a alguns fatores sociais e psicológicos, nada ainda bem definido, mais que estão relacionados a acontecimentos vitais e estresse ambiental (Smith, 2014; Gentile, 2017).

\subsection{Depressão gestacional}

Os estudos mostram que a maior incidência de depressão em mulheres começa na adolescência, quando os papéis na sociedade, as cobranças e as expectativas sofrem mudanças ou frustrações. O estresse, associado como um dos possíveis agentes etiológicos contribui para o desenvolvimento da depressão, em diversas pessoas (Field, 2017).

A maior incidência de depressão entre as mulheres pode não ser devida a uma maior vulnerabilidade, mas aos fatores estressantes especificamente vividos pelas mesmas (Erickson et al., 2017). Eles incluem as responsabilidades em casa e no trabalho, a necessidade de criar sozinha os filhos e os cuidados dispensados às crianças e aos parentes mais velhos. As expectativas sociais também são questões importantes. Discussões a respeito do papel da mulher, como a questão sobre se a mulher deve escolher entre casa e trabalho, são cruciais na avaliação do desenvolvimento de depressão (Zemestani, \& Fazeli Nikoo, 2020).

A gestação, quando desejada, raramente contribui para o desenvolvimento de depressão, e a ocorrência de aborto não parece aumentar a chance de depressão. Já as mulheres que sofrem de problemas para engravidar estão sujeitas a grande estresse e ansiedade, embora ainda não se saiba se isso aumenta o risco de depressão. A maternidade precoce (adolescência) é um fator que contribui para o desenvolvimento de depressão, devido ao grande estresse que gera (Zemestani, \& Fazeli Nikoo, 2020; Abajobir et al., 2016). 
A literatura científica indica que o período gravídico puerperal é a fase de maior prevalência de transtornos mentais na mulher, principalmente no primeiro e no terceiro trimestre de gestação e nos primeiros 30 dias de puerpério (Zemestani, \& Fazeli Nikoo, 2020). A intensidade das alterações psíquicas dependerá de fatores orgânicos, familiares, conjugais, sociais, culturais e da personalidade da gestante. Cerca de um quinto das mulheres no período gestacional e no puerpério apresenta depressão. Cabe ressaltar que a maioria dessas mulheres não é diagnosticada e tratada adequadamente (Alwa et al., 2016; Latendresse et al., 2017).

Ademais, a depressão é o transtorno mental de maior prevalência durante a gravidez e o período puerperal e está associada a fatores de risco, como antecedentes psiquiátricos, dificuldades financeiras, baixa escolaridade, gestação na adolescência, falta de suporte social, eventos estressores e história de violência doméstica. Evidências demonstram que, além de a depressão pré-natal ser mais freqüente, ela é o principal fator de risco para depressão pós-natal, sendo esta, muitas vezes, uma continuação da depressão iniciada na gestação (Gentile, 2017; Abajobir et al., 2016).

\subsection{Tratamento}

Quando o transtorno depressivo maior é diagnosticado na gestação, é um difícil dilema tanto da mulher quanto da equipe de saúde que faz seu acompanhamento pré-natal. O médico deve estabelecer e auxiliar a gestante no desenvolvimento de um plano de tratamento. Em suma, o tratamento geralmente é determinado de acordo o nível da enfermidade, quando si trata de um grau leve é recomendado psicoterapias, que incluem acompanhamento psicológico entre outros, quando o nível da depressão é de moderado a grave o tratamento mais indicado é a farmacoterapia com antidepressivos (Angelotta \& Wisner, 2017).

No que diz respeito aos antidepressivos, esses têm mostrado ser eficazes no tratamento, sendo os fármacos mais prescritos para tratar a depressão gestacional os ISRSs, os inibidores seletivo da recapção de serotonina e noradrenalina (ISRSN) e os tricíclicos (ADT). Sabendo que esses fármacos atravessam facilmente a barreira placentária, a escolha do tratamento deve ser feito com cautela. De maneira que devem ser avaliados os benefícios do tratamento medicamentoso para o controle da doença materna, e os riscos do medicamento para o embrião em desenvolvimento. Essa é uma decisão individualizada, dependendo das particularidades da doença, da possibilidade de resposta à depressão materna, da probabilidade de efeitos fetais adversos (Angelotta \& Wisner, 2017; Avram et al., 2016). Levando em consideração que tanto a doença quanto a medicação apresentam riscos para a mãe e para o feto-embrião, se a decisão de usar medicação antidepressiva durante a gravidez for tomada, a alegação é que a doença pode provocar uma ameaça maior do que o tratamento escolhido (Angelotta \& Wisner, 2017).

Em relação à terapêutica medicamentosa, a classe dos ISRS são hoje em dia mais comumente usados devido à sua eficácia estabelecida, perfil terapêutico seguro, sobreposto aos efeitos colaterais e à menor possibilidade de super dosagem. A classe é composta pela fluoxetina, paroxetina, sertralina, citalopram, escitalopram e fluvoxamina. Os ISRS inibem a recaptação da serotonina (5-HT) na fenda sináptica, dessa forma aumentando a disponibilidade da monoamina e, consequentemente, potencializando a atividade serotoninérgica. Por serem seletivos, não exercem ação sobre as catecolaminas noradrenelina e dopamina (NA e DA). Entretanto, possuem atividades anticolinérgicas, adrenérgica e histaminérgica, relacionadas com a manifestação dos efeitos adversos da classe, contudo de forma menos exacerbada que os ADT. Isso devido à baixa afinidade dos ISRS por esses receptores (Klinger \& Merlob, 2008).

A farmacocinética dos fármacos no recém-nascido não é conhecida, no entanto para a maior parte dos medicamentos o tempo de meia vida encontra-se prolongado, dependendo também do metabolismo do fármaco. Os psicofármacos entrando na corrente sanguínea, invariavelmente atravessam a barreira hemato-placentário estando o feto exposto a estes compostos (Malm et al., 2011). 


\subsection{Efeitos adversos do tratamento com ISRS sobre o feto e neonatais}

Tendo em vista todas as características abordadas, a utilização destes fármacos durante o período gestacional pode ser nocivo ao feto e o recém-nascido, além de possíveis efeitos em longo prazo sobre as crianças expostas durante a vida intrauterina. Esses efeitos estão relacionados de acordo três síndromes distintas: teratogênese, perinatal e comportamental. À medida que os antidepressivos atravessam a barreira placentária e hematoencefálica e passam para o leite materno, eles podem aumentar o nível de mediadores no feto em desenvolvimento e pode afetar adversamente o desenvolvimento funcional do cérebro. Eles representam assim um risco para neurocomportamental, emocional e cognitivo, que podem se manifestar no desenvolvimento da criança (Angelotta \& Wisner, 2017).

Sabe-se da prática clínica que as mães grávidas expostas a antidepressivos têm mais abortos espontâneos e um aumento do número de natimortos (Dubovicky et al., 2017). Os neonatos expostos a ISRS durante o terceiro trimestre também apresentam risco aumentado de desconforto respiratório, cianose, apneia, convulsões, instabilidade de temperatura, dificuldade de alimentação, vômitos, hipoglicemia, hipotonia, hipertonia, tremor, nervosismo, irritabilidade e choro constante (Grigoriadis et al., 2013; Fava et al., 2015). Esta constelação de sintomas é denominada síndrome de adaptação pós-natal (PNAS), e os sintomas são tipicamente leves por natureza (Latendresse et al., 2017). Fetos expostos a ISRSs no útero, particularmente no terceiro trimestre, apresentam risco aumentado para PNAS. Os antidepressivos serotoninérgicos mais intimamente associados à PNAS são a fluoxetina e a paroxetina, provavelmente porque esses fármacos atravessam a placenta em níveis mais altos do que os outros ISRSs (Grigoriadis et al., 2013). Há relatos de PNAS leve em 22\% dos neonatos expostos, e PNAS grave em 3\% desses recém-nascidos, levando muitos a recomendar o monitoramento clínico específico dos recém-nascidos, particularmente durante as primeiras 48 horas após o nascimento (Forsberg et al., 2014). Os sinais e sintomas podem indicar um efeito tóxico direto de ISRSs como sintomas de abstinência. As crianças que foram expostas perinatalmente a ISRSs (especialmente fluoxetina) observou-se diminuição do peso ao nascer, distúrbios neurocomportamentais e diminuição da frequência cardíaca (Rayen et al., 2011). A complexidade dessas questões é destacada, uma vez que a própria depressão pode afetar adversamente o desenvolvimento da criança. Isto é, portanto, difícil dividir o efeito da depressão em si e tratamento subsequente. Por exemplo, recém-nascidos cujas mães sofreram de depressão durante a gravidez são caracterizada pelo aumento da irritabilidade, redução da atividade e atenção, bem como falta de expressão mimética, baixo peso ao nascer, restrição no crescimento fetal e complicações pós-natais em comparação com neonatos de mães saudáveis (Kendig et al., 2017).

O uso de ISRSs durante a gravidez também está relacionada a defeitos congênitos decorrentes de sua utilização (Alwa et al., 2016; Malm et al., 2011), como por exemplo, o pé torto equinovaro congênito (Yazdy et al., 2014) - uma malformação estrutural que ocorre quando os membros inferiores não conseguem girar corretamente; isso resulta que os pés que permanecem em suas posições fetais precoce, com um ou ambos virando para dentro e para baixo. A possível via para o patogênese do pé torto pode ser através da vasoconstrição de níveis mais elevados de serotonina, dado que o pé torto tem sido hipótese de ser o resultado de uma ruptura vascular, justificando que realmente a serotonina pode ter um efeito seletivo na vasculatura uterina (Tang et al., 2019; Yazdy et al., 2014).

Grandes estudos populacionais que controlam fatores de confusão não documentaram associação entre a exposição no primeiro trimestre aos ISRSs como uma classe de medicamentos e as malformações cardíacas (Wemakor et al., 2015; Furu et al., 2015). Embora alguns estudos sem controle adequado para fatores de confusão tenham documentado um risco aumentado dfurue várias malformações congênitas e cardíacas para aqueles expostos a ISRS (Wemakor et al., 2015), vários estudos bem conduzidos não confirmaram essa associação (Furu et al., 2015; Huybrechts et al., 2014). Entretanto, uma meta-análise de 23 estudos encontrou um aumento marginalmente estatisticamente significante no risco de anomalia cardíaca específica apenas ao uso de paroxetina (Bérard et al., 2016). Os defeitos cardíacos observados eram tipicamente de natureza leve, mas incluíam anomalias no fechamento do septo cardíaco, defeitos do septo atrial e defeitos na via de saída do ventrículo direito. Esses achados 
não resultaram em nenhuma recomendação para o aumento do monitoramento, como ecografia ecocardiográfica fetal ou para aqueles expostos a ISRSs durante a gravidez, exceto, talvez, para a exposição pré-natal específica à paroxetina (Huybrechts et al., 2014; Bérard et al., 2016).

Quanto o efeito da associação possível com o transtorno do espectro do autismo (TEA), Man et al ${ }^{26}$, em sua revisão sistemática e meta-análise, concluíram que há apoio na literatura sobre um risco aumentado de autismo após a exposição prénatal aos ISRS. Assim como, Kobayashi e colaboradores chegaram a uma conclusão semelhante também em uma revisão e metaanálise de 5 casos controle e 3 estudos de coorte. Recentemente, Andalib et al. avaliaram todos os estudos publicados até junho de 2016 e concluíram que há uma associação entre exposição pré-natal a ISRSs e transtorno do espectro do autismo. Em contrapartida com esses estudos, Gentile et al. em sua revisão não encontrou nenhuma associação definida entre a exposição prénatal a ISRSs e autismo. Da mesma maneira, Kaplan et al. em sua meta-análise também não encontrou uma associação significativa entre os ISRS pré-natais e o TEA, mas observou associação com o tratamento pré-gravidez. Recentemente, um estudo de metanálise conduzida por Brown et al. não conseguiu estabelecer uma decisão quanto a tal possível associação.

Dessa forma, não há evidências suficientes para provar uma associação significativa entre ISRSs uso na gravidez e TEA nos filhos. Isso é especialmente evidente quando se controla doença psiquiátrica materna e outros fatores de confusão, incluindo o uso concomitante de outras drogas, fatores genéticos e anomalias congênitas que estão associados com TEA como comportamento. As diferenças nas conclusões das várias revisões e meta-análises podem ser explicadas pelas diferenças nos critérios de inclusão e exclusão (Gentile, 2015).

Apesar de ainda não ser totalmente esclarecida, na fisiopatologia das alterações neonatais é interessante a similaridade dos sintomas observados em recém-nascidos com as síndromes observadas em adultos: síndrome serotoninérgica, síndrome de descontinuação e síndrome colinérgica (Wang et al., 2016). A hipertensão pulmonar neonatal persistente é caracterizada por insuficiência respiratória grave e hipertensão pulmonar em recém-nascidos com mais de 34 semanas, com elevada morbidade e mortalidade, e ocorre em dois a cada 1.000 nascidos vivos. Embora o número absoluto de casos seja pequeno (um em cada 100 recém-nascidos expostos), a magnitude do risco é elevada, bem como a gravidade do quadro (Bérard et al., 2017). Estabelecida como um efeito secundário dos ISRS, uma vez que, a serotonina tem propriedades vasoconstritoras, aumentando a resistência vascular pulmonar. Assim, acumulação de altos níveis de serotonina no pulmão fetal resulta na proliferação das células do músculo liso. Em adição, a serotonina inibe a síntese do óxido nítrico, um vasodilatador que regula o tônus muscular e a reatividade tanto in útero como no pós-parto (Tafuri et al., 2017).

Sendo assim, recém-nascidos de mães tratadas com ISRSs durante o segundo semestre da gravidez deve ser cuidadosamente monitorizados nos primeiros dias após o parto para diagnosticar qualquer complicação resultante do tratamento materno: isto é, anomalias cardíacas, adaptação neonatal de hipertensão pulmonar persistente (Ornoy \& Koren, 2018). Do mesmo modo, quanto às alterações neurodesenvolvimentais, a maioria dos estudos não mostrou evidências conclusivas para um aumento do risco de neurodesenvolvimento de longo prazo após exposição intra-uterina a ISRSs. O atraso motor observado em vários estudos parece ser transitório e na maioria dos trabalhos encontrados as habilidades cognitivas e de linguagem nas crianças expostas são normais. No entanto, desde que as crianças foram examinadas em tenra idade, apesar do desenvolvimento inicial normal, efeitos negativos de habilidades de aprendizagem, capacidade de atenção e prevalência de doença mental, ainda podem ser encontradas em uma idade mais avançada, necessitando prosseguir avaliações clínicas futuras (Ornoy \& Koren, 2018).

\section{Considerações Finais}

Sendo assim, baseado nas evidências científicas fica evidente que o protocolo terapêutico com os ISRSs no tratamento da depressão gestacional gera riscos, efeitos indesejáveis e nocivos a criança. No entanto, muitas vezes o uso de ISRSs durante a gravidez é inevitável, uma vez que, a depressão pré-natal não tratada tem sido associada a tendências suicidas, incluindo a 
morte. Os estudos mostram que interromper o tratamento com ISRS em mulheres grávidas com história prévia de depressão leva à recaída na maioria das mulheres e, que a depressão não tratada durante a gravidez também demonstra ser um forte fator de risco para depressão pós-parto subsequente. Assim, em situações em que pode ser necessário usar ISRS na gravidez, a decisão de manter tal conduta terapêutica durante a gravidez deve ser avaliada contra os riscos de depressão não tratada e esta relação risco/benefício com sua adesão ao tratamento com essa classe farmacológica. Pondera-se ainda que, isso deve ser cuidadosamente discutido com o paciente e, idealmente, deve ser feito uma abordagem disciplinar em colaboração com o psiquiatra do paciente.

Recomenda-se que sejam realizados estudos clínicos randomizados robustos que padronize variáveis importantes para detecção desses efeitos adversos do uso de ISRS durante o período gestacional e que os profissionais se mantenham atualizados acerca do uso racional dos medicamentos, uma vez que novas pesquisas e publicações surgem constantemente. Negligenciar o aperfeiçoamento científico acerca desse assunto pode gerar impactos negativos e letais na saúde dos pacientes.

\section{Referências}

Abajobir, A. A., Maravilla, J. C., Alati, R., \& Najman, J. M. (2016). A systematic review and meta-analysis of the association between unintended pregnancy and perinatal depression. Journal of affective disorders, 192, 56-63. https://doi.org/10.1016/j.jad.2015.12.008.

Alwan, S., Bandoli, G., \& Chambers, C. D. (2016). Maternal use of selective serotonin-reuptake inhibitors and risk of persistent pulmonary hypertension of the newborn. Clinical pharmacology and therapeutics, 100(1), 34-41. https://doi.org/10.1002/cpt.376.

Andalib, S., Emamhadi, M. R., Yousefzadeh-Chabok, S., Shakouri, S. K., Høilund-Carlsen, P. F., Vafaee, M. S., \& Michel, T. M. (2017). Maternal SSRI exposure increases the risk of autistic offspring: A meta-analysis and systematic review. European psychiatry: the journal of the Association of European Psychiatrists, 45, 161-166. https://doi.org/10.1016/j.eurpsy.2017.06.001.

Angelotta, C., \& Wisner, K. L. (2017). Treating Depression during Pregnancy: Are We Asking the Right Questions?. Birth defects research, 109(12), 879-887. https://doi.org/10.1002/bdr2.1074.

Avram, M. J., Stika, C. S., Rasmussen-Torvik, L. J., Ciolino, J. D., Pinheiro, E., George, A. L., Jr, \& Wisner, K. L. (2016). Rationale and design for an investigation to optimize selective serotonin reuptake inhibitor treatment for pregnant women with depression. Clinical pharmacology and therapeutics, 100(1), 31-33. https://doi.org/10.1002/cpt.375.

Bérard, A., Iessa, N., Chaabane, S., Muanda, F. T., Boukhris, T., \& Zhao, J. P. (2016). The risk of major cardiac malformations associated with paroxetine use during the first trimester of pregnancy: a systematic review and meta-analysis. British journal of clinical pharmacology,81(4), 589-604. https://doi.org/10.1111/bcp.12849.

Bérard, A., Sheehy, O., Zhao, J. P., Vinet, É., Bernatsky, S., \& Abrahamowicz, M. (2017). SSRI and SNRI use during pregnancy and the risk of persistent pulmonary hypertension of the newborn. British Journal of clinical pharmacology, 83(5), 1126-1133. https://doi.org/10.1111/bcp.13194.

Brown, H. K., Hussain-Shamsy, N., Lunsky, Y., Dennis, C. E., \& Vigod, S. N. (2017). The Association Between Antenatal Exposure to Selective Seroton in Reuptake Inhibitors and Autism: A Systematic Review and Meta-Analysis. The Journal of clinical psychiatry, 78(1), e48-e58. https://doi.org/10.4088/JCP.15r10194.

Dubovicky, M., Belovicova, K., Csatlosova, K., \& Bogi, E. (2017). Risks of using SSRI / SNRI antidepressants during pregnancy and lactation. Interdisciplinary toxicology, 10(1), 30-34. https://doi.org/10.1515/intox-2017-0004.

Erickson, N. L., Gartstein, M. A., \& Dotson, J. (2017). Review of Prenatal Maternal Mental Health and the Development of Infant Temperament. Journal of obstetric, gynecologic, and neonatal nursing: JOGNN, 46(4), 588-600. https://doi.org/10.1016/j.jogn.2017.03.008.

Fava, G. A., Gatti, A., Belaise, C., Guidi, J., \& Offidani, E. (2015). Withdrawal Symptoms after Selective Serotonin Reuptake Inhibitor Discontinuation: A Systematic Review. Psychotherapy and psychosomatics, 84(2), 72-81. https://doi.org/10.1159/000370338.

Field T. (2017). Prenatal anxiety effects: A review. Infant behavior \& development, 49, 120-128. https://doi.org/10.1016/j.infbeh.2017.08.008.

Forsberg, L., Navér, L., Gustafsson, L. L., \& Wide, K. (2014). Neonatal adaptation in infants prenatally exposed to antidepressants --clinical monitoring using Neonatal Abstinence Score. PloS one, 9(11), e111327. https://doi.org/10.1371/journal.pone.0111327.

Furu, K., Kieler, H., Haglund, B., Engeland, A., Selmer, R., Stephansson, O., Valdimarsdottir, U. A., Zoega, H., Artama, M., Gissler, M., Malm, H., \& Nørgaard, M. (2015). Selective serotonin reuptake inhibitors and venlafaxine in early pregnancy and risk of birth defects: population based cohort study and sibling design. BMJ (Clinical research ed.), 350, h1798. https://doi.org/10.1136/bmj.h1798.

Gentile S. (2015). Prenatal antidepressant exposure and the risk of autism spectrum disorders in children. Are we looking at the fall of Gods?. Journal of affective disorders, 182, 132-137. https://doi.org/10.1016/j.jad.2015.04.048.

Gentile S. (2017). Untreated depression during pregnancy: Short- and long-term effects in offspring. A systematic review. Neuroscience, 342, 154-166. https://doi.org/10.1016/j.neuroscience.2015.09.001. 
Grigoriadis, S., VonderPorten, E. H., Mamisashvili, L., Eady, A., Tomlinson, G., Dennis, C. L., Koren, G., Steiner, M., Mousmanis, P., Cheung, A., \& Ross, L. E. (2013). The effect of prenatal antidepressant exposure on neonatal adaptation: a systematic review and meta-analysis. The Journal of clinical psychiatry, 74(4), e309-e320. https://doi.org/10.4088/JCP.12r07967.

Huybrechts, K. F., Hernández-Díaz, S., \& Avorn, J. (2014). Antidepressant use in pregnancy and the risk of cardiac defects. The New England journal of medicine, 371(12), 1168-1169. https://doi.org/10.1056/NEJMc1409203.

Kaplan, Y. C., Keskin-Arslan, E., Acar, S., \& Sozmen, K. (2016). Prenatal selective serotonin reuptake inhibitor use and the risk of autism spectrum disorder in children: A systematic review and meta-analysis. Reproductive toxicology (Elmsford, N.Y.), 66, 31-43. https://doi.org/10.1016/j.reprotox.2016.09.013.

Kendig, S., Keats, J. P., Hoffman, M. C., Kay, L. B., Miller, E. S., Moore Simas, T. A., Frieder, A., Hackley, B., Indman, P., Raines, C., Semenuk, K., Wisner, K. L., \& Lemieux, L. A. (2017). Consensus Bundle on Maternal Mental Health: Perinatal Depression and Anxiety. Obstetrics and gynecology, 129(3), 422-430. https://doi.org/10.1097/AOG.0000000000001902.

Klinger, G., \& Merlob, P. (2008). Selective serotonin reuptake inhibitor induced neonatal abstinence syndrome. The Israel journal of psychiatry and related sciences, 45(2), 107-113.

Kobayashi, T., Matsuyama, T., Takeuchi, M., \& Ito, S. (2016). Autism spectrum disorder and prenatal exposure to selective serotonin reuptake inhibitors: A systematic review and meta-analysis. Reproductive toxicology (Elmsford, N.Y.), 65, 170-178. https://doi.org/10.1016/j.reprotox.2016.07.016.

Latendresse, G., Elmore, C., \& Deneris, A. (2017). Selective Serotonin Reuptake Inhibitors as First-Line Antidepressant Therapy for Perinatal Depression. Journal of Midwifery \&amp; Women's Health, 62(3), 317-328. https://doi.org/10.1111/jmwh.12607.

Ludke, M. \& Andre, M. E. D. A. (2013). Pesquisa em educação: abordagens qualitativas. Ed. EPU.

Malm, H., Artama, M., Gissler, M., \& Ritvanen, A. (2011). Selective serotonin reuptake inhibitors and risk for major congenital anomalies. Obstetrics and gynecology, 118(1), 111-120. https://doi.org/10.1097/AOG.0b013e318220edcc.

Man, K. K., Tong, H. H., Wong, L. Y., Chan, E. W., Simonoff, E., \& Wong, I. C. (2015). Exposure to selective serotonin reuptake inhibitors during pregnancy and risk of autism spectrum disorder in children: a systematic review and meta-analysis of observational studies. Neuroscience and biobehavioral reviews, 49 , 82-89. https://doi.org/10.1016/j.neubiorev.2014.11.020.

Ornoy, A., \& Koren, G. (2018). Selective serotonin reuptake inhibitor use in pregnant women; pharmacogenetics, drug-drug interactions and adverse effects Expert opinion on drug metabolism \& toxicology, 14(3), 247-259. https://doi.org/10.1080/17425255.2018.1430139.

Oyebode, F., Rastogi, A., Berrisford, G., \& Coccia, F. (2012). Psychotropics in pregnancy: safety and other considerations. Pharmacology \& therapeutics, 135(1), 71-77. https://doi.org/10.1016/j.pharmthera.2012.03.008.

Pereira A. S. et al. (2018). Metodologia da pesquisa científica. UFSM.https://repositorio.ufsm.br/bitstream/handle/1/15824/Lic_Computacao_MetodologiaPesquisa-Cientifica.pdf?sequence $=1$.

Rayen, I., van den Hove, D. L., Prickaerts, J., Steinbusch, H. W., \& Pawluski, J. L. (2011). Fluoxetine during development re verses the effects of prenatal stress on depressive-like behavior and hippocampal neurogenesis in adolescence. PloS one, 6(9), e24003. https://doi.org/10.1371/journal.pone.0024003.

Smith K. (2014). Mental health: a world of depression. Nature, 515(7526), 181. https://doi.org/10.1038/515180a.

Tafuri, S., De Pasquale, V., Costagliola, A., Della Morte, R., Avallone, L., \& Pavone, L. M. (2017). Health and disease, an orchestra of three players: Serotonin, orexins, and nitric oxide. Journal of neuroscience research, 95(10), 1891-1893. https://doi.org/10.1002/jnr.24030.

Tang, X., Lu, Z., Hu, D., \& Zhong, X. (2019). Influencing factors for prenatal Stress, anxiety and depression in early pregnancy among women in Chongqing, China. Journal of affective disorders, 253, 292-302. https://doi.org/10.1016/j.jad.2019.05.003.

Wang, R. Z., Vashistha, V., Kaur, S., \& Houchens, N. W. (2016). Serotonin syndrome: Preventing, recognizing, and treating it. Cleveland Clinic journal of medicine, 83(11), 810-817. https://doi.org/10.3949/ccjm.83a.15129.

Wemakor, A., Casson, K., Garne, E., Bakker, M., Addor, M. C., Arriola, L., Gatt, M., Khoshnood, B., Klungsoyr, K., Nelen, V., O'Mahoney, M., Pierini, A., Rissmann, A., Tucker, D., Boyle, B., de Jong-van den Berg, L., \& Dolk, H. (2015). Selective serotonin reuptake inhibitor antidepressant use in first trimester pregnancy and risk of specific congenital anomalies: a European register-based study. European journal of epidemiology, 30(11), 1187-1198. https://doi.org/10.1007/s10654-015-0065-y.

Yazdy, M. M., Mitchell, A. A., Louik, C., \& Werler, M. M. (2014). Use of selective serotonin-reuptake inhibitors during pregnancy and the risk of clubfoot. Epidemiology (Cambridge, Mass.), 25(6), 859-865. https://doi.org/10.1097/EDE.0000000000000157.

Zemestani, M., \& Fazeli Nikoo, Z. (2020). Effectiveness of mindfulness-based cognitive therapy for comorbid depression and anxiety in pregnancy: a randomized controlled trial. Archives of women's mental health, 23(2), 207-214. https://doi.org/10.1007/s00737-019-00962-8. 